Claremont Colleges

Scholarship@ Claremont

Pitzer Faculty Publications and Research

Pitzer Faculty Scholarship

7-1-2003

\title{
Trying to Fix the Development in Evolutionary Developmental Psychology
}

David S. Moore

Pitzer College

\section{Recommended Citation}

Moore, David S. "Trying to Fix the Development in Evolutionary Developmental Psychology." Review of The Origins of Human Nature: Evolutionary Developmental Psychology, by David F. Bjorklund and Anthony D. Pellegrini. The American Journal of Psychology 116.2 (2003): 299-307. Print. for inclusion in Pitzer Faculty Publications and Research by an authorized administrator of Scholarship @ Claremont. For more information, please contact scholarship@cuc.claremont.edu. 


\section{The content of this open-access post-print article is the same as that contained in the published article with the following reference:}

Moore, D. S. (2003, Summer). Trying to fix the development in Evolutionary

Developmental Psychology. The American Journal of Psychology, 299 - 307.

Trying to Fix the Development in

Evolutionary Developmental Psychology

If we agree for the moment that there is such a thing as "human nature," we immediately encounter an extraordinarily thorny question: where does our nature come from? This question drives David Bjorklund and Anthony Pellegrini's new book, The Origins of Human Nature: Evolutionary Developmental Psychology. The question is so challenging, in part, because human nature reflects at least two conceptually distinct processes, evolution and development. The former, which operates across generations, allows for the continued existence of characteristics that permitted survival and reproduction in our ancestors; the latter, which operates during an individual's lifetime, contributes to the appearance of all of our characteristics. The problem facing students of human nature is how to understand the relationship between these processes, and how they contribute to the appearance of our traits.

It is not difficult to see how developmental processes impact the appearance of our traits, because these processes operate in real-time; they are proximal causes of traits, and as such, can be studied - theoretically, at least — in the same way that chemical and physical events can be studied. Evolutionary processes, in contrast, are not so easily studied. As distal causes, their effects must be mediated by other more proximal agents, and as causes that are sometimes rooted in the ancient past and that take generations to be manifested, they can rarely be observed directly. To make matters even more 
complicated, the proximal agent that evolutionary processes use to produce their effects is none other than development itself. Untangling this spaghetti, not surprisingly, is no easy task.

Bjorklund and Pellegrini's book, written for psychologists but valuable for students of evolution or development in general, valiantly takes on this challenge. The authors' stated goal is to provide "a developmental perspective for evolutionary psychologists and an evolutionary perspective for developmental psychologists" (p. 4). This is a laudable goal, as evolutionary psychologists need to understand development (development is, after all, the proximal agent of evolution) and developmentalists need to be familiar with evolution (psychological theories that are irreconcilable with evolutionary theory are of dubious value, since behaviors, thoughts, and feelings are products of biology, and evolutionary theory underlies all current understandings of biology). The Origins of Human Nature covers an extremely important corpus of data, and Bjorklund and Pellegrini persevere in the face of difficult, outstanding problems.

The major obstacle to a truly integrative theory of evolutionary developmental psychology has been an apparently inherent incompatibility between the perspectives of contemporary evolutionary and developmental psychologists. As Bjorklund and Pellegrini observe, "the primary reason for this misfit... was the lack of a specific model of how evolved and thus genetically transmitted psychological mechanisms get expressed" (p. $x$ ). Herein we see the problem: among developmentalists who have struggled intently with these issues, a consensus has emerged that there can be no such things as "genetically transmitted psychological mechanisms," because genes are simply unable to carry all of the information needed to express such mechanisms. As a result, 
some of these developmentalists — whose position Bjorklund and Pellegrini call "the developmental systems approach"- think that evolutionary psychology and developmental psychology, as currently configured, might be intrinsically in conflict with one another, and therefore beyond integration (R. Lickliter, personal communication, April, 2002). Nonetheless, while professing adherence to the tenets of the developmental systems approach themselves, Bjorklund and Pellegrini strive in their book to integrate aspects of these two branches of psychology.

The first five chapters of the book give a theoretical framework for a new discipline of evolutionary developmental psychology. Chapters 6 through 10, in contrast, focus on particular topics within the discipline. The phenomena examined in the latter chapters are considered mostly from an evolutionary psychological perspective; the authors typically address the adaptive function of each one, focusing on a characteristic's contribution to survival and reproduction in the Pleistocene "environment of evolutionary adaptedness." Additionally, most of the subsections in these chapters contain systematic examinations of available data on non-human primates. In the final chapter (11), the authors flesh out the following basic principles of the field:

1. Evolutionary Developmental Psychology involves the expression of evolved, epigenetic programs. [This principle lies at the heart of the authors' integration of developmental and evolutionary psychology, because it implies — correctly — that all evolved characteristics undergo development.]

2. An extended childhood is needed in which to learn the complexities of human social communities. 
3. Many aspects of childhood serve as preparations for adulthood and were selected over the course of evolution.

4. Some characteristics of infants and children were selected to serve an adaptive function at specific times in development and not as preparations for adulthood.

5. Many, but not all, evolved psychological mechanisms are domain-specific in nature.

6. Evolved mechanisms are not always adaptive for contemporary people.

These ideas appear regularly throughout the book, and are quite familiar by the time they are summarized in the epilogue.

After beginning in Chapter 1 with an overview of their subject and the structure of their book, Bjorklund and Pellegrini offer in Chapters 2 and 3 historical accounts of Darwin's theory of evolution, early conceptions of the relationship between evolution and development, and the emergence of evolutionary psychology. Also in these chapters is a very good — and much needed, for most developmentalists — review of the fossil record on which current understandings of human morphological evolution are based. The authors' depiction of the Pleistocene environment in which Homo sapiens is thought to have evolved is particularly evocative. In addition, these chapters present an accurate overview of the developmental systems approach, and some of the $20^{\text {th }}$ century theories of evolution that might be compatible with it (including those offered by developmental psychologist James Mark Baldwin, developmental biologist Conrad H. Waddington, and comparative developmental psychobiologist Gilbert Gottlieb). Along the way, Bjorklund and Pellegrini discuss adaptationist thinking (which characterizes evolutionary psychology and which stresses traits' functions), the strivings of contemporary theorists 
to establish a useful definition of the word "innate," and various attempts to use evolutionary theory to understand the origin of individual differences (e.g., behavior genetics and social Darwinism). Among the more interesting ideas considered include ontogenetic adaptations (traits that help organisms adapt to their circumstances during particular phases of development only), and heterochrony (an evolutionary process involving the alteration of developmental rates from generation to generation). Developmental psychologists will be well served by considering the topics examined in these chapters; the authors have presented this information in an accessible way, providing valuable assistance to their colleagues.

In Chapter 4, Bjorklund and Pellegrini examine the importance of developmental timing, and the importance of slow development in humans in particular. The heterochronic process now suspected to be largely responsible for human evolution is called neoteny; it involves a slowing of development hypothesized to have occurred as our primate ancestors evolved into Homo sapiens. Some of the effects of this hypothesized developmental slowing - including the evolution of our characteristically large and "plastic" brains, and the emergence of the complex structures that characterize our societies - appear to have contributed to our success in our niche. As in Chapters 2 and 3, the authors convey how investigations of evolution can draw attention to developmental phenomena and how studies of development in modern humans and other primates can illuminate aspects of evolution. In particular, the authors propose that cognitive immaturity can be adaptive, and they buttress their suggestion with supportive data and strong arguments. While some of the sections of this chapter are necessarily speculative, they are nevertheless interesting and important. 
Chapter 5 presents a catalogue of dichotomous constructs developed by

psychologists in the last few decades; it considers each in turn as it relates to evolutionary developmental psychology. Specifically, Bjorklund and Pellegrini consider implicit versus explicit cognition and memory, domain-general versus domain-specific cognitive capacities, immediate versus deferred benefits of children's cognition, and cognitive abilities that are "biologically primary" ("evolved to deal with problems faced by our ancestors") versus "biologically secondary" ("instilled in children by their cultures to deal with 'new' ecological problems"). The discussions in this chapter are essential reading insofar as subsequent chapters rely heavily on these distinctions in their exposition; the section on implicit and explicit cognition and memory is particularly well written.

In their chapter on prepared learning (Chapter 6), Bjorklund and Pellegrini write about characteristics with which we are born, or that appear later in development but seemingly independently of specific experiences. Some of these characteristics include infants' perceptual preferences, their "knowledge" of the behavior of physical objects, their alleged basic quantitative skills, and their abilities to acquire language and spatial cognition proficiency. In their chapter on social cognition (Chapter 7), the authors consider social learning (including mimicry, emulation, and imitative, instructed, and collaborative "cultural learning"), the development in early childhood of an understanding that other people have mental states corresponding to beliefs, intentions, and desires, and the development of social reasoning. As they do throughout the book, the authors focus here on sex differences in cognition and behavior, and on the possible evolutionary origins of these differences. Chapter 8 begins with a recounting of parental 
investment theory—not much changed, as Bjorklund and Pellegrini tell it, since Trivers first proposed it in 1971 — and continues by examining parent/child, sibling, and stepparent/step-child relationships from this perspective. Chapter 9 reports on the development of social interactions, relationships, and small peer group dynamics. In keeping with their evolutionary perspective, the authors recount in this chapter Hamilton's (1964) theory of inclusive fitness and Maynard Smith's (1972) concept of evolutionarily stable strategies; likewise, in keeping with their interest in comparative data, they explain their inclination to view social structures in terms of dominance relationships. Their section on relationships begins by considering the origins of attachments in infants and toddlers, and their section on interactions ends by considering the origins of violent behavior in adolescents.

Before presenting their chapter 11 epilogue with its six "basic principles" of evolutionary developmental psychology, the authors offer a final substantive chapter on the development of play. It is clear from this chapter that Bjorklund and Pellegrini are experts on this subject, and that it lends itself to both developmental and evolutionary analyses. As such, play is an excellent domain in which to apply many of the concepts discussed in earlier chapters. Here, we find treatments of sex differences in play, of the function of play both as an ontogenetic adaptation and as a generator of benefits deferred until adulthood, of the appearance of play in industrialized societies, hunter-gatherer societies, and in non-human animals, and of the effects of slow development on the amount of play experienced in a lifetime.

Because good psychological theories must be compatible with theories of biology and because any truly comprehensive theory of biology will be characterized by an 
integrated understanding of evolution and development, Bjorklund and Pellegrini's efforts to generate an "overarching metatheory" that integrates evolutionary and developmental psychology are well placed ${ }^{1}$. Unfortunately, in spite of the high quality of their finished volume, it appears that the time might not yet be ripe for an integration of these two branches of psychology. The authors' goals are praiseworthy, their labors are nearly heroic, and there are moments when their simultaneous focus on development and evolution seems to yield genuine integration. Nevertheless, their approach does not seem to have achieved the full-scale integration they hoped it would.

The major obstacle to such an integration continues to be the genetic determinism embedded in evolutionary psychology, the denials of this determinism by both traditional evolutionary psychologists and by Bjorklund and Pellegrini notwithstanding. Consider the quotation the authors use from developmental neuroscientist Mark Johnson:

"Since it has become evident that genes interact with their environment at all levels, including the molecular, there is no aspect of development that can be said to be strictly "genetic," that is, exclusively a product of information contained in the genes" (p. 34).

In spite of this conclusion, three sentences later, Bjorklund and Pellegrini write, "information-processing biases derive from genetically specified features of neurons [as well as from other factors]." But if none of our characteristics are "exclusively a product of information contained in the genes," how can there be "genetically specified features"

\footnotetext{
${ }^{1}$ Note, though, that the final sentence of their book is seriously overstated: it cannot be the case that the theory of evolution "should also be the basis of modern psychology..." (p. 341), because not all psychological characteristics have evolved. In fact, whereas an evolutionary perspective is necessary to understand the appearance of some characteristics, it is not sufficient for understanding the appearance of any; this follows from the fact that a developmental perspective is necessary for understanding the appearance of all traits (although it is sufficient to explain the appearance of only some). For this reason, it
} 
of neurons? Similarly, when the authors write, “... some innately influenced behaviors can be highly canalized... whereas others can be less specifically directed by the genome" (p. 67), they imply that highly canalized behaviors are "specifically directed by the genome," a proposal that is simply not compatible with what is known about gene functioning.

Even when evolutionary psychologists do not specifically mention genes, their hypotheses often implicitly suggest a reliance on genetic determinism (or at very least, an unjustified belief in an extreme canalization of human behavioral development). Consider, for example, Bjorklund and Pellegrini's following two statements: "[Because] modern women...possess the same evolved psychology as their ancestral grandmothers and contemporary women in traditional societies...[they] are not immune to the thoughts and feelings that [unhealthy] children may invoke in them and, particularly under economically and socially trying circumstances, they may behave in socially unacceptable ways [by not investing in those children]" (p. 228229).

“...human males have inherited a psychology that was adapted to different conditions in which risky competition, on the average, resulted in increased inclusive fitness" (p. 275-277).

Obviously, the first of these statements endeavors to avoid genetic determinism by positing a role for social circumstances in the production of behavior. Nonetheless, if the 
experiential factors important for the development of these behaviors are not available today exactly as they were millions of years ago, then contemporary people could possess the same psychology as their ancestors only if that psychology were genetically determined. As we will see in a moment, this conclusion follows from Bjorklund and Pellegrini's acknowledgement that "What are inherited are developmental systems..." (p. $80)$.

According to the developmental systems approach advocated by the authors, people inherit genes and environments from their ancestors, not full-blown, developed psychologies. Full-blown characteristics emerge during development as a result of continuous and bi-directional transactions among inherited environmental and genetic resources. Therefore, modern people could be expected to have the same psychological characteristics as their ancestors only if the experiential factors that contribute to the development of those characteristics are available in modern environments exactly as they were in ancestral environments (of course, various genetic factors would need to be unchanged as well). But in fact, our developmental experiences today are dramatically different than were those of our ancestors. As the authors note, “...cultural changes over the past 10,000 years have drastically affected the way humans behave and develop. Cultural influences such as nutrition and health care directly affect brain growth and development, as do aspects of material culture, including modern tools, methods of communication, and formal education. Humans did not evolve in a world anything like the one most people experience today..." (p. 8). 
Consequently, we are unlikely to "inherit" our ancestors' psychologies. Claims by evolutionary psychologists that we do necessarily imply either reliance on genetic determinism — which in the current case would be unwitting, as the authors are at pains to avoid such determinism — or on an untenable belief that experiential factors required to develop these psychologies have remained unchanged since the Pleistocene epoch. Especially now that we know how remarkably "plastic" the human brain is (Kaas, 1991; Merzenich, 1998), there is no reason to believe that psychological development is so highly canalized as to be unresponsive to the changes in human developmental environments that have characterized the last million years. If some modern women fail to invest in their unhealthy children, or if some modern men engage in risky competition, it is entirely possible that that is because such behaviors make sense to them in the present, and not necessarily because such behaviors improved fitness in our distant ancestors.

Given the authors' explicit endorsement of the developmental systems approach, it is important to ask (as they do in their epilogue), "what, then, is one to make of the evolved psychological mechanisms postulated by evolutionary psychologists?" Their answer is that "such mechanisms can be thought of as genetically coded 'messages' or 'rules of thumb' that, following epigenetic rules, interact with the environment (again, broadly defined) over time to produce behavior" (p. 336). This represents a bold attempt to make sense of evolutionary psychologists' postulations while maintaining sight of the fact that development is a fundamentally epigenetic process. Unfortunately, the idea that genes contain coded "messages" or "rules of thumb" has not withstood empirical scrutiny (Oyama, 2000). Instead, the information necessary for development is distributed across 
genetic and non-genetic developmental resources available to growing organisms (Griffiths \& Gray, 1994; Lickliter \& Berry, 1990). Moreover, neither type of developmental resource controls the other; genes collaborate with non-genetic factors to produce developmental outcomes (Gottlieb, Wahlsten, \& Lickliter, 1998). Genes, if they even exist as stable entities — which is not at all clear at present (Keller, 2000; Moore, 2002) — do not contain coded "messages" that are implemented according to preestablished epigenetic rules (Oyama, 2000); evolved psychological mechanisms, whatever else they might be, cannot be thought of as genetically coded "rules of thumb." This line of argument leads to a related concern about Bjorklund and Pellegrini's conclusion that human infants are born with "epigenetic programs that have evolved over eons..." (p. 336). It is misleading to characterize the epigenetic events that occur during normal development as "programs" insofar as this word—as borrowed from computer science - implies the existence of predetermined functions specified by an intelligent designer. Instead, the theoretical perspective most consistent with the developmental systems approach is Dynamic Systems Theory (Thelen \& Smith, 1998); this perspective holds that development occurs in real-time, and does not progress inexorably toward a predetermined end-state. Because this perspective is ascendant among some developmentalists, a reference to it might have been helpful.

While it remains for evolutionary psychologists to work out the mechanism by which certain psychological characteristics might be faithfully passed from generation to generation, an even more difficult problem involves finding a way to identify evolved psychological characteristics in the first place. This problem arises when one adopts a developmental systems approach, because this approach eliminates the possibility of 
distinguishing evolved and un-evolved traits based on the developmental mechanisms responsible for their appearance. Whereas early evolutionary theories permitted the fantasy that evolved traits are inherited via strictly genetic mechanisms and that the development of un-evolved traits, in contrast, requires environmental input, the developmental systems approach recognizes that all characteristics develop as a function of the same epigenetic mechanisms; there are no such things as traits that appear without environmental input (broadly construed). But if developmental mechanisms can no longer help us distinguish evolved from un-evolved traits, what can?

One might be tempted to argue that evolved traits appear invariably, in all normal, species-typical environments (i.e., their development is highly canalized). Unfortunately, this approach will not allow us to distinguish evolved and un-evolved traits, because some traits that evolutionary psychologists would consider un-evolved also appear in all normal environments; an example might be the wrinkles that appear in older people's skin. Alternatively, one might argue that evolved traits have an adaptive function, whereas un-evolved traits do not. However, once again, some traits that evolutionary psychologists would consider un-evolved do have adaptive functions; a fear of guns could very well be adaptive in the $21^{\text {st }}$ century, but evolutionary psychologists would deny that such fears could have evolved in the short time since the invention of firearms. Furthermore, it turns out that traits that have been subjected to natural selection need not necessarily be adaptive after all. Consider Bjorklund and Pellegrini's following three sentences:

“...how can new cognitive abilities appear if they have not gone through the sieve of natural selection?" (p. 113). 
"What infants come into the world with are processing biases and constraints - products of natural selection - that serve as the foundation for developing a human mind" (p. 191).

"Evolutionary developmental psychology assumes that not only are the behaviors and cognitions that characterize adults the product of natural selection, but so are characteristics of children's behaviors and minds" (p. $335)$.

Each of these statements reflects confusion over the true source of biological and behavioral novelties: such innovations are always created via developmental processes, not evolutionary ones. In fact, natural selection per se is not a creative force, and so can never produce new behaviors, cognitive abilities, "processing biases and constraints," or any other characteristic; natural selection can only remove characteristics that interfere, ultimately, with reproduction. Consequently, natural selection yields both adaptive and neutral characteristics, which are otherwise indistinguishable. The upshot is that traits that have "gone through the sieve of natural selection"-characteristics that first appeared during an ancestor's development, and that have been faithfully transmitted through the generations because they do not hinder reproduction - do not necessarily have an adaptive function; some of these traits are merely "neutral." Although Bjorklund and Pellegrini routinely refer in their book to "evolved psychological characteristics," there appears to be no way to determine if these traits really did evolve, because such traits 
currently seem indistinguishable from un-evolved traits. Any successful integration of evolutionary and developmental psychology will necessarily address this problem.

In the end, while some of the characteristics of children's psychologies are likely to have evolved, studying the evolution of these traits ought not be the primary goal of an intellectual endeavor called "evolutionary developmental psychology." Using an evolutionary psychology perspective to examine children's traits will not generate "evolutionary developmental psychology," because evolutionary psychologists, to date, have not attempted to account for the emergence of these traits in development. When an approach leads to the conclusion that a childhood trait has evolved but does not offer an account of the emergence of the trait in development, that approach should be called evolutionary child psychology, not evolutionary developmental psychology. The appropriate goal of the latter endeavor should be an understanding of how developmental processes are transmitted and modified across generations. While Bjorklund and Pellegrini clearly understand this criterion—in their words, “...evolutionary developmental psychology is not simply evolutionary psychology applied to infants and children" (p. 4) —only some of what they present in their book is free of the nondevelopmentalism that characterizes canonical evolutionary psychology; other parts still read like evolutionary psychology applied to juveniles.

The foregoing concerns notwithstanding, The Origins of Human Nature fills a gap in the contemporary psychological literature, as it attempts to bridge two of today's fastest-growing branches of psychology. While addressing one of the most important questions imaginable and not shying away from the difficult issues it presents, Bjorklund and Pellegrini manage to offer a remarkably current and somewhat integrated review of 
several significant bodies of data. An interdisciplinary work of considerable value, The Origins of Human Nature raises many thought-provoking questions, even as it missteps by acceding to some of the flaws inherent in traditional evolutionary psychology. At the very least, the authors have produced a work that draws attention both to the need for an integration of evolutionary and developmental psychology, and to the long road that still lies before the integrators; in the process, they have provided both needed information and enjoyment for their readers. 


\section{References}

Bjorklund, D. F. \& Pellegrini, A. D. (2002). The origins of human nature: Evolutionary developmental psychology. Washington, D.C.: American Psychological Association.

Gottlieb, G., Wahlsten, D., \& Lickliter, R. (1998). The significance of biology for human development: A developmental psychobiological systems view. In W. Damon (Series Ed.) \& R. M. Lerner (Vol. Ed.), Handbook of child psychology: Vol. 1. Theoretical models of human development ( $5^{\text {th }}$ ed., pp. 233-234). New York: Wiley.

Griffiths, P. E. \& Gray, R. D. (1994). Developmental systems and evolutionary explanation. The Journal of Philosophy, XCI, 277-304.

Hamilton, W. D. (1964). The genetical theory of social behavior. Journal of Theoretical Biology, 7, $1-52$.

Kaas, J. H. (1991). Plasticity of sensory and motor maps in adult mammals. Annual Review of Neuroscience, 14, 137-167.

Keller, E. F. (2000). The century of the gene. Cambridge, MA: Harvard University Press.

Lickliter, R. \& Berry, T. D. (1990). The phylogeny fallacy: Developmental psychology's misapplication of evolutionary theory. Developmental Review, 10, 348364.

Maynard Smith, J. (1972). On evolution. Edinburgh, Scotland: Edinburgh University Press.

Merzenich, M. (1998). Long-term change of mind. Science, 282, 1062- 1063. 
Moore, D. S. (2002). The dependent gene: The fallacy of nature vs. nurture. New York: Times Books/Henry Holt.

Oyama, S. (2000). The ontogeny of information: Developmental systems and evolution ( $2^{\text {nd }}$ ed.). Durham, NC: Duke University Press.

Thelen, E. \& Smith, L. B. (1998). Dynamic systems theories. In W. Damon (Series Ed.) \& R. M. Lerner (Vol. Ed.), Handbook of child psychology: Vol. 1. Theoretical models of human development ( $5^{\text {th }}$ ed., pp. 563-634). New York: Wiley.

Trivers, R. L. (1971). The evolution of reciprocal altruism. Quarterly Review of Biology, 46, $35-57$. 